\title{
Power Macro-Modeling Using an Iterative LS-SVM Method
}

\author{
António Gusmão, Luis Miguel Silveira, and José Monteiro \\ INESC-ID / IST, TU Lisbon, Lisboa, Portugal \\ \{antoniog, lms , jcm\}@algos.inesc-id.pt
}

\begin{abstract}
We investigate the use of support vector machines (SVMs) to determine simpler and better fit power macromodels of functional units for high-level power estimation. The basic approach is first to obtain the power consumption of the module for a large number of points in the input signal space. Least-Squares SVMs are then used to compute the best model to fit this set of points. We have performed extensive experiments in order to determine the best parameters for the kernels.

We propose a new method for power macromodeling of functional units for high-level power estimation based on Least-Squares Support Vector Machines (LS-SVM). Our method improves the already good modeling capabilities of the basic LS-SVM method in two ways. First, a modified norm is used that is able to take into account the weight of each input for global power consumption in the computation of the kernels. Second, an iterative method is proposed where new data-points are selectively added as support-vectors to increase the generalization of the model.

The macromodels obtained compare favorably with those obtained using industry standard table models, providing not only excellent accuracy on average (close to $1 \%$ error), but more importantly, thanks to our proposed modified kernels, we were able to reduce the maximum error to values close to $10 \%$.
\end{abstract}

Keywords: Power Estimation, Macromodel, SVM, Pruning, Norm.

\section{Introduction}

Electronic systems have become pervasive in our daily lives, work environments and even our social habits. Their design and verification prior to fabrication are challenging tasks due to inherent size and complexity. Rising costs of design and market pressure for fast delivery of error-free systems drive the need for reliable verification. A common approach towards easing the design process and enabling verification is to replace large and complex design blocks by smaller, more abstract macromodels that accurately represent relevant characteristics of the system. The resulting macromodeled system can then be verified and design exploration can be accomplished, leading to better, more efficient designs, with lower costs and added features. 
Power consumption has become one of the most important parameters in the design of VLSI circuits and accurate power estimation a requisite of any design exploration framework and verification environment. Many high-level power estimation tools have been proposed before to enable the evaluation of different architectures at early stage of design [8. The general approach is to use power macromodels for each functional unit. These macromodels are obtained in a precharacterization phase, stored in a library for later use and represent the power dissipation of the unit as a function of its input statistics.

Kernel methods provide a powerful and unified framework for pattern discovery, motivating algorithms that can act on general types of data and look for general types of relations (e.g. rankings, classifications, regressions, clusters) [16]. The application areas range from neural networks and pattern recognition to machine learning and data mining. In this paper, we investigate the use of learning algorithms, in particular support vector machines (SVMs), to determine simpler and better fit power macromodels. The basic approach is first to obtain the power consumption of the module for a large number of points in the input signal space. Least-Squares SVMs (LS-SVM) are then used to compute the best model to fit these set of points. The statistics for each of the module's output signals can be computed in a similar manner, thus providing a means of propagating the switching probabilities through the circuit. We have performed extensive experiments in order to analyze the possible kernels which are the basis of the SVM formulation and to determine the best parameters for these kernels.

Our proposed methodology improves the already good modeling capabilities of the basic LS-SVM method in two ways. In general, kernels treat every dimension uniformly. In the problem at hand, each input to the functional module defines a dimension for the kernel (although not necessarily so after optimizations, as discussed in Section 5.4). It is well-known that not all inputs have the same impact on the power consumption of a module. We propose a modification to the basic RBF kernel that takes into account a measure of the contribution of each input for the power consumption in the computation of the kernels. Secondly, an iterative method is proposed where new data-points are selectively added as support-vectors to better generalize the model

We present results that confirm the excellent modeling capabilities of the kernel-based methods. The macromodels obtained provide not only excellent accuracy on average (all below $2 \%$ average error and close to $1 \%$ on average), but, more importantly, thanks to our modified kernels, we were able to reduce the maximum error to values close to $10 \%$. As we will show, such low error rates compare favorably with those obtained using standard table-based models.

The paper is organized as follows. In Section 2, we review previous work on power analysis techniques at the RT level and provide some background on kernel methods. Section 3 discusses the kernel parameters and their set up. The proposed optimization techniques, namely the modified norm and the iterative process, are described in Section 4. The implementation details of the power macromodeling process are described in Section 5. We present our results in Section 6 and draw conclusions and future work in Section 7. 


\section{Related Work}

\subsection{Power Macro-Modeling}

There has been a fair amount of work on generating models for power dissipation at higher levels of abstraction. Top-down approaches have been proposed in [9] and [10. They are both based on the concept of entropy and their focus is to derive implementation-independent measures of the signal activity in the circuit. A number of assumptions are made in both [9] and [10] on how to propagate the entropy of the inputs through the circuits. These methods can be very efficient, though given all the required approximations and the fact that they ignore issues such as glitching implies that these techniques are not very accurate.

Our method follows a bottom-up approach (for a survey, see [8]), where the model is obtained from an actual circuit implementation. This offers the best level of accuracy. These methods build their models from data points that consist of a power value for the circuit, under some input conditions. From this set of data points, different strategies exist for generating a model that not only fits these data points, but offers the best possible generalization ability.

Lookup tables have been successfully proposed [3]. N-dimensional tables have been used, where each dimension represents an input parameter. Several strategies exist for reducing the number of dimensions and for interpolating among table points. Alternatively, regression can be used to compute the coefficients of an expression [1211/17. A combination of both of these methods has also been proposed [3. Models for specialized functions, such as arithmetic units, use different expressions for different inputs, namely depending on whether they correspond to the most or the least significant bits [6]. Using models tailored to specialized functions, however, requires very specific knowledge of the problem which restricts the applicability of the technique. Table interpolation or regression implicitly assumes a polynomial or spline representation and may require a large number of coefficients if the underlying surface is somewhat nonlinear. SVMs are quite useful in such a context as they can readily adapt to the data at hand, even if it exhibits strong nonlinearities. Therefore, much sparser approximants can be used while retaining or even improving on the accuracy.

We believe the model we propose, based on LS-SVMs, is more robust than previously proposed approaches: it is generic, systematic, and uses an underlying methodology with properties that have been proven both theoretically and in practice in many different fields. Our results demonstrate just that.

\section{$2.2 \quad$ LS-SVMs}

Consider a general problem where we are given $N$ input/output data points, $\left\{\mathbf{x}_{k}, z_{k}\right\}_{k=1}^{N} \in \mathbb{R}^{p} \times \mathbb{R}$. These data points follow an unknown function $z(\mathbf{x})=$ $m(\mathbf{x})+e(\mathbf{x})$, where $m(\mathbf{x})$ is the target function we wish to estimate and $e(\mathbf{x})$ is a sampling error. Support Vector Machines (SVMs) are a method of obtaining $y(\mathbf{x})$, an estimate of $m(\mathbf{x})$, from the given data set, referred to as training set. SVMs achieve regression by nonlinearly mapping the input space into a higher 
dimensional feature space where a linear approximant hyperplane can be found. This is implicitly made by the use of a kernel function.

A version of a SVM for regression was proposed by Vapnik el al 16. This method is called support vector regression (SVR). The model produced by SVR only depends on a subset of the training data, because the cost function for building the model ignores any training data that are close (within a threshold $\varepsilon)$ to the model prediction. The relevant data points form a set of support vectors and they immediately lead to a sparse representation. On the other hand, computing the model is a Quadratic Programming (QP) problem. To simplify this QP problem, Least Squares Support Vector Machines were introduced [15]. In both methods the model is:

$$
y(\mathbf{x})=\mathbf{w}^{T} \varphi(\mathbf{x})+b
$$

The $\varphi(\mathbf{x})$ mapping is usually a non-linear function that transforms the data into a higher dimensional feature space, and is weighted by $\mathbf{w}$. Constant $b$ is the bias term.

LS-SVM corresponds to solving the following constrained optimization problem:

$$
\min _{\mathbf{w}} J=\frac{1}{2} \mathbf{w}^{T} \mathbf{w}+C \frac{1}{2} \sum_{k=1}^{N} e_{k}^{2} \quad \text { s.t. } \quad z_{k}=\mathbf{w}^{T} \varphi\left(\mathbf{x}_{k}\right)+b+e_{k}
$$

The $\mathbf{w}^{T} \mathbf{w}$ term stands for minimizing the length of the weight vector, while the $\mathrm{C}$ constant is the trade-off parameter between the complexity of the representation and the minimization of training data errors. The number of training samples, known as support vectors, is given by $N$.

Using Lagrange multipliers, in order to transform the problem into an unconstrained optimization problem, gives:

$$
L=\frac{1}{2} \mathbf{w}^{T} \mathbf{w}+C \frac{1}{2} \sum_{k=1}^{N} e_{k}^{2}-\sum_{k=1}^{N} \alpha_{k}\left[\mathbf{w}^{T} \varphi\left(\mathbf{x}_{k}\right)+b+e_{k}-z_{k}\right]
$$

which is guaranteed to have a global minimum when:

$$
\frac{\partial L}{\partial \mathbf{w}}=0 ; \frac{\partial L}{\partial b}=0 ; \frac{\partial L}{\partial e_{k}}=0 ; \frac{\partial L}{\partial \alpha_{k}}=0,
$$

resulting in the following linear system of equations:

$$
\left[\begin{array}{cc}
0 & \mathbf{1}^{T} \\
\mathbf{1} & \boldsymbol{\Omega}+C^{-1} \boldsymbol{I}
\end{array}\right]\left[\begin{array}{l}
b \\
\boldsymbol{\alpha}
\end{array}\right]=\left[\begin{array}{l}
0 \\
\mathbf{z}
\end{array}\right]
$$

where $\boldsymbol{\Omega}$ is the kernel matrix, $\Omega_{k l}=\Psi\left(x_{k}, x_{l}\right)=\varphi\left(x_{k}\right) \cdot \varphi\left(x_{l}\right), k, l=1, \ldots, N$, and $\Psi$ is the kernel function.

The resulting LS-SVM is given by:

$$
y(\mathbf{x})=\sum_{k=1}^{N} \boldsymbol{\alpha}_{k} \Psi\left(\mathbf{x}, \mathbf{x}_{k}\right)+b
$$


Table 1. Common kernels

\begin{tabular}{|c|c|}
\hline Linear: & $\Psi\left(\mathbf{x}_{i}, \mathbf{x}_{j}\right)=\mathbf{x}_{i}^{T} \mathbf{x}_{j}$ \\
\hline Polynomial: & $\Psi\left(\mathbf{x}_{i}, \mathbf{x}_{j}\right)=\left(\mathbf{x}_{i}^{T} \mathbf{x}_{j}+\theta\right)^{n}$ \\
\hline Exponential (RBF): & $\Psi\left(\mathbf{x}_{i}, \mathbf{x}_{j}\right)=\exp \left(\frac{-\left\|\mathbf{x}_{i}-\mathbf{x}_{j}\right\|^{2}}{\sigma^{2}}\right)$ \\
\hline Hyperbolic Tangent: & $\Psi\left(\mathbf{x}_{i}, \mathbf{x}_{j}\right)=\tanh \left(\phi \mathbf{x}_{i}^{T} \mathbf{x}_{j}+\theta\right)$ \\
\hline
\end{tabular}

The simplicity of (5) is not without a cost. While SVMs have a built in way of selecting the most significant data points from their training set, LS-SVMs do not. Sparseness is lost due to the usage of all the data points as support vectors (a large $N$ ). This adds a new complexity to the problem. It becomes necessary to judiciously select the training datapoints used to effectively cover the input space.

There are many kernels from which to choose from, some of which are shown in Table 1. In this work, we will focus exclusively on the RBF kernel since it has good empirical results and has a nice smooth behavior.

\section{Kernel Tuning}

The regression process is not totally automated, since there are a number of parameters that need to be selected, namely $C$ (see (4)), $\sigma$ (see Table 11) and the number of support vectors, $N$. We also have an option as to which kernel to use, however the exponential RBF kernel (Table1) has been reported to perform particularly well under LS-SVM [12. The analysis and results presented in the remainder of this paper were all obtained with this kernel.

\subsection{Parameter $C$}

As referred in Section 2.2, $C$ is a constant that permits a tradeoff between the training error and the smoothness of the model. The training error in sample $k$ is given by $e_{k}=\frac{\alpha_{k}}{C}$, indicating that larger values of $C$ force the estimator to reduce the training errors. We have computed the errors obtained using different values of $C$, maintaining all remaining parameters constant $(\sigma=2$ and with 2,000 $\mathrm{SVs}$ ). These errors are obtained on a set of datapoints disjoint from the training set, and which is called the test set.

We use the following error functions to provide some insight into LS-SVM model's performance: 
Relative error: $E_{R}=\left\{\frac{\left.\left|z_{k}-y\left(\mathbf{x}_{k}\right)\right|\right\}}{z_{k}}\right\}_{k=1}^{N}$

Average relative error: $E_{1}=\frac{1}{N} \sum_{k=1}^{N} E_{R_{k}}$

Maximum relative error: $E_{2}=\max _{k} E_{R_{k}}$

Fraction below 10\%: $E_{3}=\frac{\left|\left\{a \in E_{R}: a<10 \%\right\}\right|}{N}$

Ultimately we aim to achieve an $E_{3}$ of $100 \%$ and an $E_{1}$ smaller than $1 \%$. Although the error values are not bounded, $E_{2}$ is a good representation of the worst-case scenario.

The graphs in Figure 1 show the test error variation with $C$ averaged over all the benchmark circuits (given in Table 3). For all the circuits the behavior was similar so it is safe to derive conclusions from the average results. We can observe that the error decreases as $C$ increases, but for values above $C=10^{4}$ the error remains almost constant. Hence, both training and test errors benefit from larger $C$.

A new experiment was devised to show that the optimum $C$ depends on the noise present in the data points. Consider the earlier data set $\left\{\mathbf{x}_{k}, z_{k}\right\}$ with added noise: $z_{k}^{\prime}=(1+\epsilon) z_{k}$ where $\epsilon$ follows a uniform distribution between $[-e ; e]$.

Figure 2 shows the test errors for noisy data with maximum noise $e=10 \%$. It becomes clear that the value of $C$ depends on the quality of the data set. We have set $C=10^{4}$ since it is approximately optimal for the noiseless case and it is still good when there are small errors present.
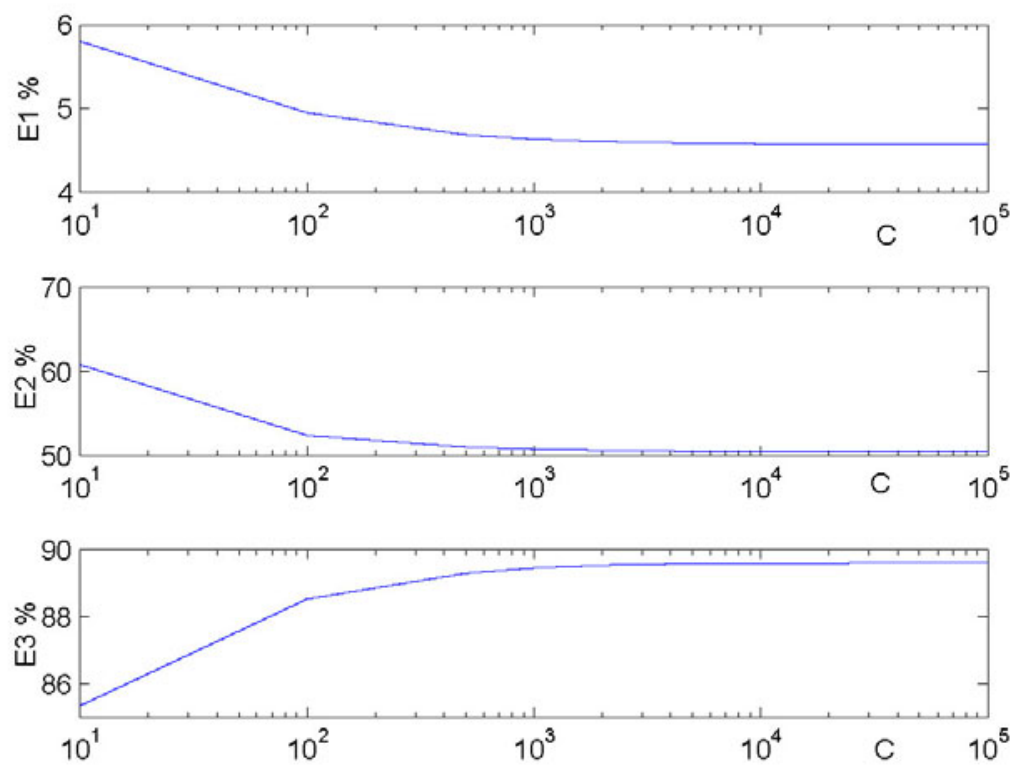

Fig. 1. Error variation with parameter $C$ 

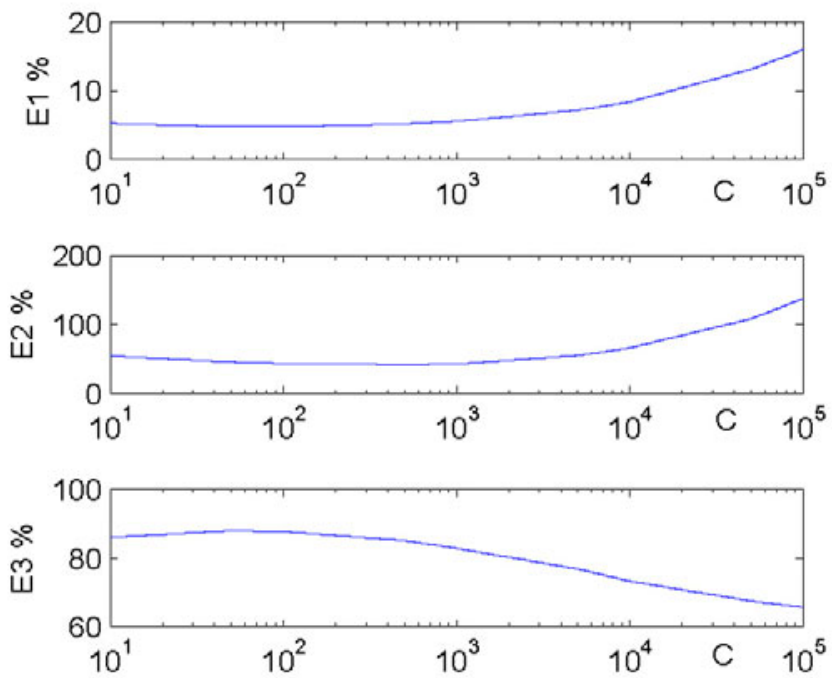

Fig. 2. Error variation with parameter $C$ for noisy data (10\%)

\subsection{Parameter $\sigma$}

In the exponential RBF kernel (Table 1), $\sigma$ represents a width factor. If it is large, then the influence of each support vector (SV) spreads, smoothing the solution. If $\sigma$ is small, each SV has a small influence over the space around it, which reduces the information the model has over all the input space. At the
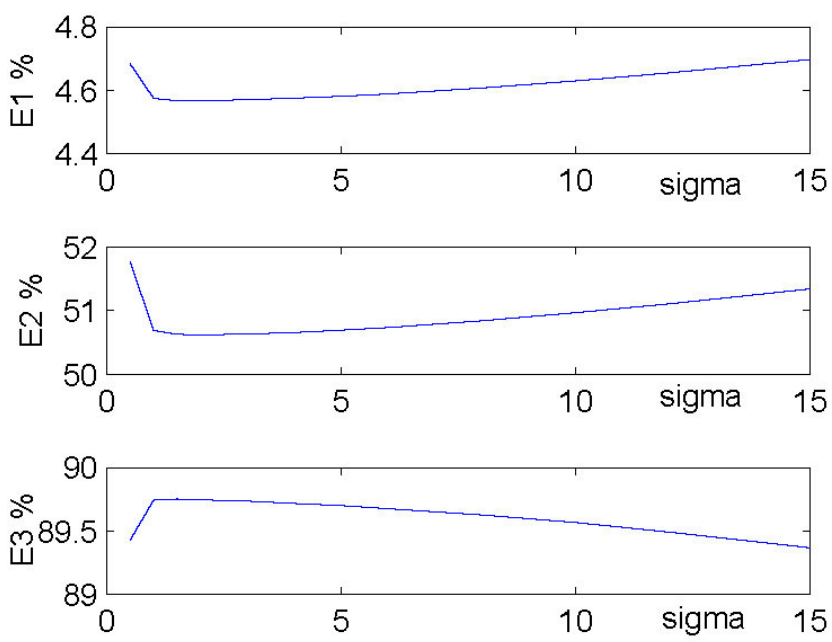

Fig. 3. Errors for different values of $\sigma$ 
extremes, if $\sigma \rightarrow \infty$ we obtain a constant function, and if $\sigma \rightarrow 0$ our model is only able to estimate input $\mathbf{x}$ equal to its SVs, having null generalization ability. Training error is then inversely proportional to $\sigma$ values.Figure 3 presents the error obtained for different values of $\sigma$ on test data $\left(C=10^{4}\right.$, obtained above, and the same 2,000 number of SVs were used). Test results indicate that a value of $\sigma=1.1$ produces the best overall results (the training error is negligibly small for this $\sigma$ ). If we are to assume the same value of sigma for all the circuits, then this would be the value to use for $\sigma$.

Figure 4 shows how each support vector contributes to the final solution in an artificial two-dimensional problem, for two different values of $\sigma, \sigma=1$ and $\sigma=0.02$, respectively the top (a, b) and bottom (c, d) graphs. The graphs on the right (a, c) present a separate curve for each support vector and the graphs on the left $(\mathrm{a}, \mathrm{d})$ present the resulting model (summation of all components).

Three important conclusions are drawn:

1. for large values of $\sigma$ the resulting surfaces are very smooth, and that might make it impossible to follow a steep function.

2. small values of $\sigma$ allow more complex and steep surfaces, but unless all input space is well covered, bad generalization will occur.

3. as there is a single $\sigma$ for all SVs, the only degree of freedom LS-SVMs have is the selection of the $\boldsymbol{\alpha}$ weights of each SV, which serves as a scaling factor to the respective component curve (the bias term, $b$, is one more degree of freedom, but it is only an added constant).
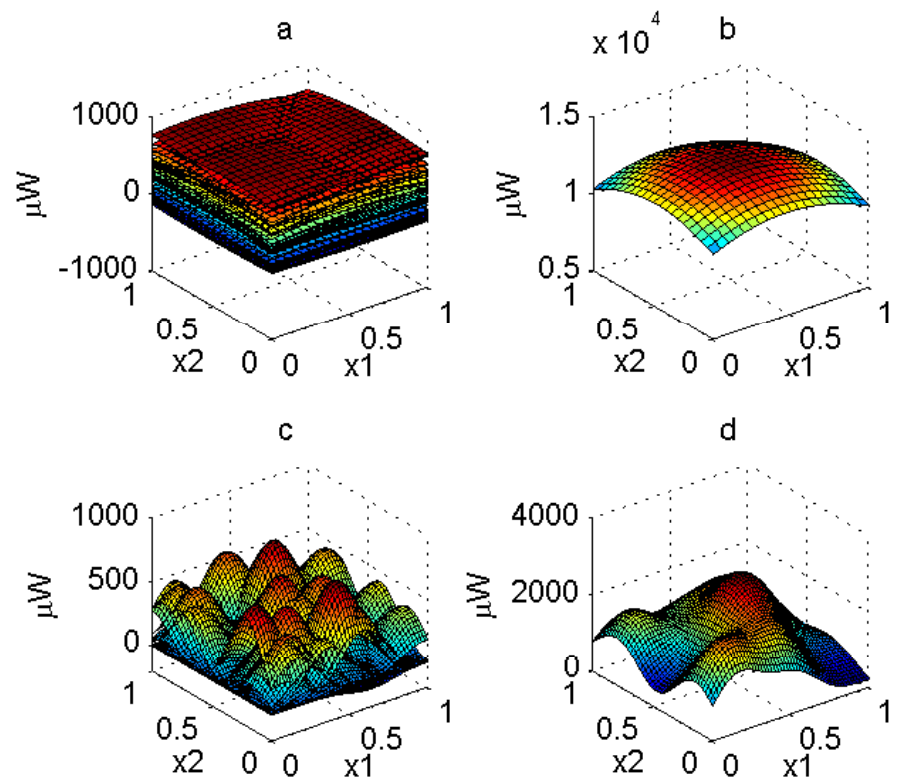

Fig. 4. Power surfaces for: a) $\sigma=1$, components; b) $\sigma=1$, sum; c) $\sigma=0.02$, components; d) $\sigma=0.02$, sum 

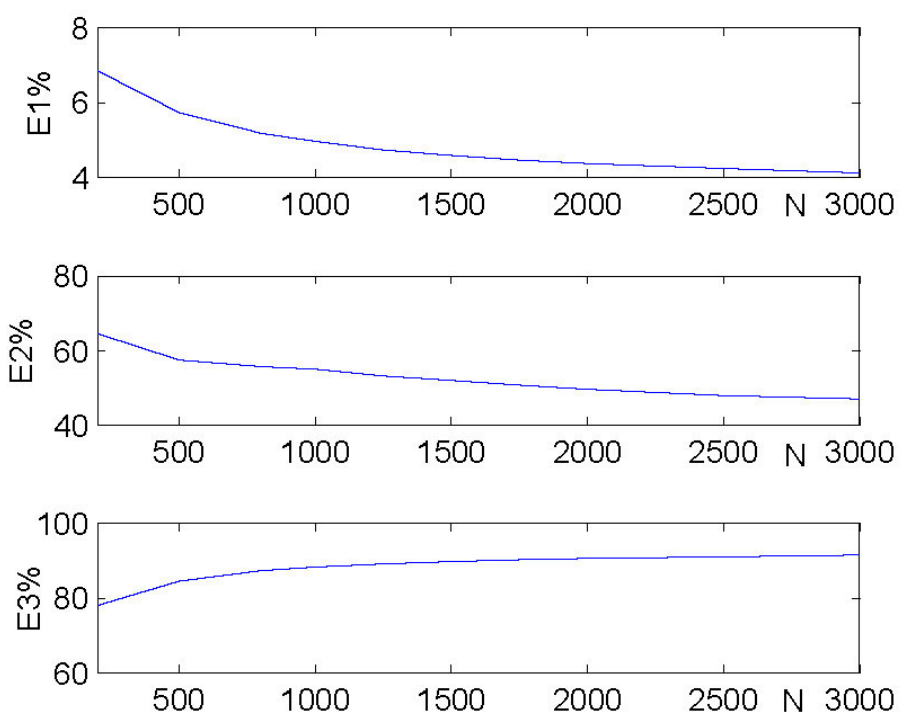

Fig. 5. Error dependence on the number of support vectors

Issues 1 and 2 could be solved by choosing an 'optimal' $\sigma$ value, but it would still imply that all dimensions of the input space have the same behavior (all smooth or all steep). We propose in Section 4.2 an iterative approach to automatically determine the $\sigma$ value for a given problem instance. Issue 3 seems to be a major restriction of the models using a RBF kernel. The model output, $y(\mathbf{x})$, is computed based on the distance between the input vector $\mathbf{x}$ and all of the model SVs. We address this issue in Section 4.1.

\subsection{Number of Support Vectors}

To determine the number of SVs, we performed similar experiments with increasing size of the training data set (Figure 5). As expected, errors decrease when the size of the training set increases. Since having more SVs translates linearly to the size of the model and its computation time, it is necessary to achieve a tradeoff between model complexity, the number of SVs, and model accuracy. One should be aware that the number of SVs will depend on the shape of power surface (steep functions need more SVs) and the $\sigma$ value used (smaller sigmas imply more SVs). This motivates the use of an iterative algorithm to add SVs to the training set when there is a need to do so, as described in Section 4.2

\section{Model Optimization}

In this section, we present the improvements we propose to the LS-SVM method. 


\subsection{Weighted Norm}

Consider as an example a particular situation where:

1. $\mathbf{x} \in R^{p}$ are samples of the $p$ inputs of a functional unit in a logic circuit.

2. one of them, $x_{r}$, has a huge effect on the power dissipated (for instance, a RESET signal).

3. we have a trained LS-SVM model with $N$ support vectors, $N>p$.

4. two test vectors are given, $\mathbf{x}_{1}$ and $\mathbf{x}_{2}$, which are exactly the same except in their $x_{r}$ component.

Since the only information the model uses to differentiate the output values $y\left(\mathbf{x}_{1}\right)$ and $y\left(\mathbf{x}_{2}\right)$ is their distance to all of the $N$ support vectors, it is natural to assume that for $p \gg 1$ their distances to the $N$ SVs would be almost the same which results in $y\left(\mathbf{x}_{1}\right) \approx y\left(\mathbf{x}_{2}\right)$. We know that their real outputs are very different and so the LS-SVM model can never give a good prediction of these values. A possible solution to this problem would be to get a very large number of SVS that would cover that critical input space where $x_{r}$ varies. This is very costly and would prove to be extremely difficult since that means that there would be many SV close to each other, implying small $\sigma$ s and, thus, poor generalization ability.

To solve this problem, we introduce our proposed modification to the RBF kernel which spawns from a simple adaptation of the distance measure used. By adding weights, $\boldsymbol{\beta}$, to each dimension of the input space, we add significantly more flexibility to the LS-SVM training procedure. The norm becomes

$$
\left\|\mathbf{x}_{i}-\mathbf{x}_{j}\right\|=\sqrt{\frac{\sum_{l=1}^{p} \beta_{l}\left(x_{i_{l}}-x_{j_{l}}\right)^{2}}{\sum_{l=1}^{p} \beta_{l}}}
$$

Parameters $\boldsymbol{\beta}$ must be computed before training the model. In the case of power macromodeling, a formal method to obtain $\boldsymbol{\beta}$ would be to resort to the Shannon expansion for each circuit input [13. In Section 5.2, we present a more expedite method.

Note that a similar weighing of the different dimensions can be applied to other kernels (Table 1), where the internal product needs to be modified to use a different coefficient for each dimension.

\subsection{Iterative Process}

As stated before, the best number of support vectors to use and the value of the $\sigma$ parameter are interdependent. We propose an iterative method to automatically determine these parameters so that we maximize the generalization of the LSSVM-based power macromodel.

The standard method of computing the kernel parameters is simply to solve the linear system given in Equation 4, using a set of data points $\left\{\mathbf{x}_{k}, z_{k}\right\}_{k=1}^{N}$, 
the training set. The model obtained can then be evaluated against a different and disjoint set of datapoints, the test set. From the obtained errors in each of these sets, we can conclude:

1. large errors on the test set might be due to two reasons: the 'area' of the input space where samples have large test errors is badly covered by the training set; or the kernel function is excessively local, which means that the influence of each SV is limited to a very small 'area' around it.

2. large errors on the training set indicate that the kernel function is smoother than it should be, not having enough flexibility to approximate steep surfaces.

To address these issues an extension to the training procedure was devised consisting of an iterative addition of SVs.

The iterative method developed starts from a given LS-SVM model and uses the following three sets:

- the final test set, with data points which will only be used to evaluate the final model (hence, after the iterative process has finished)

- an initial training set of size $M_{T}$ of the given model.

- a validation set of size $M_{V}$, which can be much larger than $M_{T}$

The following steps are repeated while user specifications have not been met, namely that the average relative error, $E_{1}$, and the maximum relative error, $E_{2}$, on the validation set are smaller than given thresholds $T_{E_{1}}$ and $T_{E_{2}}$ :

1. to increase the generalization, we move $k$ data points with the largest errors from the validation set into the training set.

2. if the error on the training set exceeded specifications, we reduce parameter $\sigma$ by $s$. As discussed in the previous section, the $\sigma$ parameter defines how local or global the impact of each support vector is. Hence, by reducing this value by multiplying it by a factor $s, s<1$, the error is reduced on the training set. This may potentially lead to worse generalization, which is compensated by the extra data points that are moved to the training set. Nevertheless, $\sigma$ should not be greatly reduced between iterations.

The initial $\sigma$ value should be relatively large to start with a very smooth solution and steepening it only as much as necessary by reducing $\sigma$. Parameters $k$ and $s$ define the granularity of the process. Larger values will reduce the number of iterations, but may lead to a larger model. The size of the initial model and respective training set should be relatively small to give room for the addition of the more problematic data points in the validation set, but, at the same time, large enough to give reasonably good predictions. In our experiments, we start from a model of size 500 obtained from applying (7) to a training set of size 2,000 .

\section{Implementation}

In this section, we describe the methodology for computing the power macromodel. We start by presenting how we obtain the data points used as 
the training set, then we describe the experiments we performed to tune the kernel parameters, and finally we discuss the size of the model and methods to reduce it.

\subsection{Input Space Analysis}

To generate the desired black-box macromodel it is necessary to obtain a set of data points to be plugged into the LS-SVM, $\left\{\mathbf{x}_{k}, z_{k}\right\}$. To analyze the performance of the LS-SVM method for power estimation, we need data points where $z_{k}$ represents the power dissipation of a circuit under inputs $\mathbf{x}_{k}$. For this purpose, we can either use experimental values obtained from actual circuit measurements, or values computed by a simulator. Naturally, the accuracy of the model will be directly related to the quality of the data points.

Note that there is some flexibility in terms of what $\mathbf{x}_{k}$ represents. In this work, we are using the switching probability of each of the $p$ inputs to the functional unit, hence a vector of size $p$ with values between 0 and 1 . Alternatively, we could aggregate all inputs and have their distribution probability (for example, in the case a set of bits represent some numerical value). Additionally, if specific information is available regarding joint probability distributions, it can be used to bias the choice of data points.

We should also observe that $z_{k}$ can represent both static or dynamic power, or total power. The results we present in the next section were obtained from a logic simulator which only accounts for dynamic power. Yet, the results should easily extrapolate for static, and hence, total power.

As data points used during training represent the total knowledge LS-SVMs have for model construction, their selection method is of crucial importance. For each circuit, the objective is to generate $N$ vectors $\mathbf{x}$ of size $p$ with values within $[0,1]$. To effectively cover the input space three distributions were tested:

1. UNIFORM, $100 \%$ follow an uniform distribution between 0 and 1 .

2. Norm, $100 \%$ follow a normal distribution with 0.5 mean and a chosen variance $\gamma$ (every value not contained in the $[0,1]$ interval is resampled).

3. UNMIX, a mix of 1 and 2, 50\% follow an uniform distribution and $50 \%$ follow a normal distribution.

Table 2. Comparison of input distributions

\begin{tabular}{|c||r|c|c|}
\hline Distribution & $\mathrm{E}_{1}$ & $\mathrm{E}_{2}$ & $\mathrm{E}_{3}$ \\
\hline \hline UnIFORM & 1.68 & 23.1 & 99.7 \\
\hline UNMix $(\gamma=0.1)$ & 1.53 & 24.8 & 99.5 \\
\hline UNMix $(\gamma=0.3)$ & 1.5 & 24.2 & 99.6 \\
\hline NORM $(\gamma=0.1)$ & 2.24 & 34.2 & 97.7 \\
\hline NORM $(\gamma=0.3)$ & 1.57 & 26.5 & 99.4 \\
\hline
\end{tabular}


For the purpose of testing the generalization ability of our model, test sets of 8,000 points were constructed using equal quantities of each of the 3 methods presented, for each of the benchmark circuits. In Table 2 the errors defined earlier (Section 3.1) are shown, averaged over all the circuits.

We conclude that the difference in error performance between the tested methods is small, yet the UNMIX distribution seems to lead to slightly smaller error values. Adding to that, in practice, circuit input probabilities should be around 0.5 so we opted to use UNMIX with a variance $\gamma=0.3$ to get a better representation in that area.

\subsection{Computing the Input Weights}

In order to gauge the relative importance of each input to the functional unit in terms of the impact in power consumption, we performed a set of experiments where we set all other inputs to a fixed value and measure the power as we change the value of the input under evaluation. Naturally, the results obtained depend on the values assigned to the other inputs. Ideally one would want to check the impact on power consumption of a single input for all possible combinations of the other inputs. Since this is impractical, we randomly sample 20 different combinations of values for the remaining inputs.

From these experiments, we compute the power range for each input, as the difference between the maximum and minimum power values. We use this value directly as the weight for this input in the computation of the modified norm.

\subsection{Model Size}

From Equation 5, we know that evaluating the LS-SVM model to compute the power requires the sum of $N$ elements, and each element contains a norm (Equation (6) which is a sum of $p$ elements. Hence, the model requires $O(N p)$ operations to compute $y(\mathbf{x})$. Computing the model's output is much faster than simulating the circuit. On a $3 \mathrm{GHz}$ AMD64 it took no more than 10 seconds to estimate 10,000 outputs for any of the tested circuits.

It terms of memory, we need to store: $N$ SVs, each of size $p ; N \alpha$ values; and the constant $b$. Hence, the non modified kernel has $O(N(p+1)+1)=O(N p)$ memory complexity. Our modified kernel adds $p$ input weight coefficients $(\beta)$, which has a negligible impact on memory usage.

If we use the float data type to store these values (usually 4 bytes long), a model of a circuit with 200 inputs $(p=200)$ and $2,000 \mathrm{SVs}(N=2000)$ will need $(2000 \times(200+1)+200+1) \times 4=1.53 M B$ of storage space. It is affordable, but still expensive. Next, we discuss methods to reduce this size.

\subsection{Model Pruning}

There are two parameters that define the model size, $N$ and $p$. There are methods that reduce $N$ by searching for "redundant" SVs, i.e., SVs whose removal has minimal impact on the error [5]. 
In our work, we apply one of the methods prescribed in [5]. In each step the algorithm solves the linear system (eq. 4) and removes the least important SV which is the $\mathbf{x}_{k}$ with the smallest $\mathrm{d}\left(\mathbf{x}_{k}\right)$ :

$$
d\left(\mathbf{x}_{k}\right)=[A . \Delta \mathbf{x}]_{k+1}=\frac{\alpha_{k}}{\left[A^{-1}\right]_{k+1}}
$$

where $A$ is the square matrix from Equation 4. This is an expensive method but has a good model-reduction to model-error ratio. For a more detailed description we refer the reader to the original paper.

Additionally, we investigate the reduction of $p$, which amounts to a form of feature selection. The process is to simply remove input dimensions with the lowest $\beta$ values, as these are the ones which have less impact in defining the power characteristics of the circuit. Starting from lower $\beta$, we remove dimensions until the sum of removed betas (normalized) is below a user specified threshold $T \in[0,1]$. Figure 6 shows the increase in error with the increase of $T$.

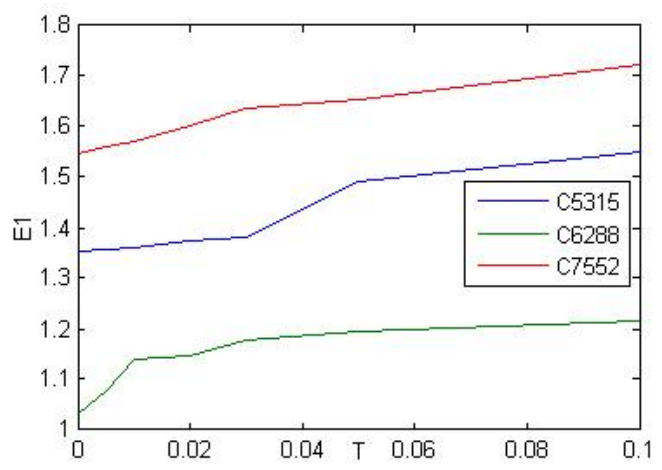

Fig. 6. Error increase with the increase of $T$

\section{Results}

In Table 3 we present results for circuits of the ISCAS benchmark set, obtained under different methods. Here we compare several modifications of the basic LS-SVM method with state of the art table lookup methods. In the table, $E_{1}$ represents average error, $E_{2}$ maximum error and $E_{3}$ estimates with error below $10 \%$. Under "Usual Norm" we give the performance of the base LS-SVM models, computed using $C=10^{4}, \sigma=1.1$ (Section [3) and from training sets of $N=$ 2,000 samples, generated as described in Section 5.1. Weighted norm tests are shown in columns "Weighted Norm", under the same conditions. Under "Iter. Weighted Norm" are the results after the iterative process, where $N$ indicates how many support vectors were used. The results reported by [3] are given under "4D Tables". Finally, under "Pruned" are the results after applying the pruning technique described in Section 5.4 using a threshold value of $T=0.08$. 
Table 3. LS-SVM test results

\begin{tabular}{|c|c|c|c|c|c|c|c|c|c|c|c|c|c|c|}
\hline \multirow{2}{*}{ Circuit } & \multicolumn{2}{|c|}{ Info } & \multicolumn{3}{|c|}{ Usual Norm } & \multicolumn{3}{|c|}{ Weighted Norm } & \multicolumn{4}{|c|}{ Iter. Weighted Norm } & \multicolumn{2}{|c|}{ 4D Table } \\
\hline & Ins & Nodes & $E_{1}$ & $E_{2}$ & $E_{3}$ & $E_{1}$ & $E_{2}$ & $E_{3}$ & $N$ & $E_{1}$ & $E_{2}$ & $E_{3}$ & $E_{1}$ & $E_{2}$ \\
\hline & 4 & & 5 & 21.3 & 3.0 & & & & 0 & 0.5 & & & .9 & 6 \\
\hline 800 & 60 & 383 & 3 & 69.2 & 5.8 & .6 & 16.0 & 99.0 & 1000 & 1.4 & 17. & 100 & .6 & 14.0 \\
\hline & & & 1 & .2 & 3.9 & & & & 00 & 0.5 & & & & 10.0 \\
\hline & 33 & & 4.9 & 7 & 1.6 & 0 & 0.8 & 99 & 95 & 1.1 & 9.8 & & .7 & 15.7 \\
\hline 267 & 33 & 93 & 3 & 36.2 & 7.4 & 3 & 9.2 & 100 & 000 & 1.2 & 7 . & 100 & 2.2 & 10.2 \\
\hline 540 & 50 & 1669 & 9 & 50.4 & 5.4 & .1 & 14.0 & 99.9 & 740 & 1.2 & 11.6 & 99.9 & 3.2 & 15.6 \\
\hline & 78 & 2307 & .0 & 21.5 & 96.5 & & & 100 & 00 & 1.1 & 5. & 100 & 2.1 & 12.2 \\
\hline 6288 & 32 & 2406 & 3.9 & 44.7 & 95.7 & 0 & 9.1 & 100 & 500 & 0.6 & 5.4 & 100 & 2.2 & 17.4 \\
\hline 50 & 207 & 3512 & 4.4 & 27.1 & 6.2 & 3 & 13.4 & 99.9 & 1000 & 1.3 & 11.2 & 99.9 & 2.7 & 14.3 \\
\hline \multicolumn{3}{|c|}{ average: } & 4.7 & 36.6 & 91.7 & 1.0 & 9.73 & 99 & 726 & 1.0 & 16 & 99.98 & 3.1 & 4 \\
\hline
\end{tabular}

Table 4. LS-SVM test results with pruning

\begin{tabular}{|c|c|c|c|c|c|c|}
\hline Circuit & Ins & Nodes & Ins & $E_{1}$ & $E_{2}$ & $E_{3}$ \\
\hline C499 & 41 & 202 & 33 & 1.0 & 6.0 & 100 \\
\hline $\mathrm{C} 880$ & 60 & 383 & 39 & 1.7 & 16.6 & 99.8 \\
\hline C1355 & 41 & 546 & 33 & 0.9 & 5.9 & 100 \\
\hline C1908 & 33 & 880 & 28 & 1.3 & 11.1 & 100 \\
\hline C2670 & 233 & 1193 & 107 & 1.3 & 8.7 & 100 \\
\hline C3540 & 50 & 1669 & 29 & 1.2 & 15.1 & 99.9 \\
\hline C5315 & 178 & 2307 & 94 & 1.1 & 7.0 & 100 \\
\hline C6288 & 32 & 2406 & 29 & 1.0 & 10.7 & 100 \\
\hline C7552 & 207 & 3512 & 124 & 1.3 & 13.1 & 100 \\
\hline \multicolumn{3}{|c|}{ average: } & & 1.2 & 10.5 & 99.97 \\
\hline
\end{tabular}

Several interesting observations can be made from the results presented in Table 3. First, we note that the results obtained with the original kernel were already very good, with average error $E_{1}$ below $5 \%$. However, maximum error $E_{2}$ can be large, with circuit C880 presenting an error close to $70 \%$. The use of the proposed weighted norm is by itself very effective in reducing error. Its impact is especially visible on the maximum error, which on average reduces to just under 10\%, with circuit C880 still presenting the largest maximum error, but reducing from $70 \%$ to $16 \%$. On average, the $E_{1}$ comes to under $1 \%$ and only $0.06 \%$ of the samples have error above $10 \%\left(E_{3}\right)$ !

The next set of results demonstrates that the iterative process allows for significantly smaller models sizes, while practically maintaining the error levels. Whereas for the previous results a constant number of 2,000 support vectors is used in the model, the iterative process finds equally good solutions with only 726 support vectors on average, providing models almost $3 \times$ more compact. 
Table 4 presents the results obtained after pruning. Note that the model size can still be effectively reduced using the proposed pruning method, without significant effect on the error level. For larger examples, the number of inputs effectively used in the model are close to half of the original circuit, leading a model half the size and half the evaluation time.

Moreover, we can observe that the results obtained clearly surpass existing state-of-the-art methods based on table lookup, both in terms of average $\left(E_{1}\right)$ and maximum $\left(E_{2}\right)$ errors.

\section{Conclusions}

Our experiments show that LS-SVM are a viable method for the generation of power macromodels. Modifying the basic RBF kernel so that it takes into account the impact of different circuit inputs on dissipated power proved to result in a huge improvement in model accuracy. It also opened doors to a new approach to model size reduction based on input dimension weights and the use of the weighted norm on other kernels. Further work involves applying kernels composed of more than one element [14.

Acknowledgments. This research was supported in part by the Portuguese FCT under program POCTI.

\section{References}

1. Bogliolo, A., Benini, L., De Micheli, G.: Regression-Based RTL Power Modeling. ACM Transactions on Design Automation of Electronic Systems 5(3), 337-372 (2000)

2. Chen, Z., Roy, K.: A power macromodeling technique based on power sensitivity. In: Proceedings of Design Automation Conference, pp. 678-683 (June 1998)

3. Gupta, S., Najm, F.: Power Modeling for High-level Power Estimation. IEEE Transactions on Very Large Scale Integration (VLSI) Systems 8, 18-29 (2000)

4. Gusmao, A., Silveira, L.M., Monteiro, J.: Parameter tuning in svm-based power macro-modeling. In: Quality of Electronic Design (ISQED 2009), pp. 135-140 (March 2009)

5. Hoegaerts, L., Suykens, J.A.K., Vandewalle, J., De Moor, B.: Primal space sparse kernel partial least squares regression for large scale problems. In: Proceedings of 2004 IEEE International Joint Conference on Neural Networks, vol. 1, p. 563 (July 2004)

6. Jochens, G., Kruse, L., Schmidt, E., Nebel, W.: A new parameterizable power macro-model for datapath components. In: Proceedings of Design, Automation and Test in Europe Conference and Exhibition 1999, pp. 29-36 (1999)

7. Landman, P.E., Rabaey, J.M.: Architectural power analysis: The dual bit type method. IEEE Transactions on Very Large Scale Integration (VLSI) Systems 3(2), 173-187 (1995)

8. Macii, E., Pedram, M., Somenzi, F.: High-level Power Modeling, Estimation, and Optimization. IEEE Transaction on Computer-Aided Design of Integrated Circuits and Systems, 1061-1079 (1998) 
9. Marculescu, D., Marculescu, R., Pedram, M.: Information Theoretic Measures of Energy Consumption at Register Transfer Level. In: Proceedings of the International Symposium on Low Power Electronics and Design, pp. 81-86 (April 1995)

10. Najm, F.: Towards a High-Level Power Estimation Capability. In: Proceedings of the International Symposium on Low Power Electronics and Design, pp. 87-92 (April 1995)

11. Raghunathan, A., Dey, S., Jha, N.K.: High-level Macro-modeling and Estimation Techniques for Switching Activity and Power Consumption. IEEE Transactions on Very Large Scale Integration (VLSI) Systems 11(4), 538-557 (2003)

12. Shang, W., Zhao, S., Shen, Y.: Application of LSSVM with AGA Optimizing Parameters to Nonlinear Modeling of SRM. In: 3rd IEEE Conference on Industrial Electronics and Applications, pp. 775-780 (June 2008)

13. Shannon, C.: The Synthesis of Two-Terminal Switching Circuits. Bell System Technical Journal 28, 59-98 (1949)

14. Smits, G., Jordaan, E.: Improved SVM Regression using Mixtures of Kernels. In: Proceedings of the 2002 International Joint Conference on Neural Networks, IJCNN 2002, vol. 3, pp. 2785-2790 (2002)

15. Suykens, J., Van Gestel, T., De Brabanter, J., De Moor, B., Vandewalle, J.: Least Squares Support Vector Machines. World Scientific Pub., Singapore (2002)

16. Vapnik, V.: Statistical Learning Theory. John Wiley \& Sons, Chichester (1998)

17. Wu, Q., Qiu, Q., Pedram, M., Ding, C.-S.: Cycle-accurate macro-models for rtlevel power analysis. IEEE Transactions on Very Large Scale Integration (VLSI) Systems 6(4), 520-528 (1998) 